\title{
Obesity Genetics
}

\section{Is the adiposity-associated FTO gene variant related to all-cause mortality independent of adiposity? Meta-analysis of data from 169,551 Caucasian adults}

\author{
E. Zimmermann ${ }^{1 \star}$, L. H. Ängquist ${ }^{1 \star}$, S. S. Mirza², J. H. Zhao ${ }^{3}$, D. I. Chasmann, ${ }^{4,5}$, K. Fischer $^{6}$, Q. Qi $^{7}$, \\ A. V. Smith ${ }^{8,9}$, M. Thinggaard ${ }^{10}$, M. N. Jarczok ${ }^{11}$, M. A. Nalls ${ }^{12}$, S. Trompet ${ }^{13,14}$, N. J. Timpson ${ }^{15}$, \\ B. Schmidtt ${ }^{16}$, A. U. Jackson ${ }^{17}$, L. P. Lyytikäinen ${ }^{18,19}$, N. Verweij ${ }^{20}$, M. Mueller-Nurasyid ${ }^{21,22,23,24}$, \\ M. Vikström ${ }^{25}$, P. Marques-Vidal26, A. Wong ${ }^{27}$, K. Meidtner ${ }^{28,29}$, R. P. Middelberg ${ }^{30}$, R. J. Strawbridge ${ }^{31}$, \\ L. Christiansen ${ }^{10}$, The FTO-Mortality Collaborating Group ${ }^{\dagger}$, K. O. Kyvik ${ }^{32}$, A. Hamsten ${ }^{31}$, T. Jääskeläinen ${ }^{33}$, \\ A. Tjønneland ${ }^{34}$, J. G. Eriksson ${ }^{35,36,37,38}$, J. B. Whitfield ${ }^{30}$, H. Boeing ${ }^{29}$, R. Hardy²7, P. Vollenweider ${ }^{26}$, \\ K. Leander ${ }^{25}$, A. Peters ${ }^{24,39}$, P. van der Harst ${ }^{20,40,41}$, M. Kumari4 ${ }^{42,43}$, T. Lehtimäki ${ }^{18,19}$, A. Meirhaeghe ${ }^{44}$, \\ J. Tuomilehto $45,46,47,48$, K.-H. Jöcke ${ }^{16}$, Y. Ben-Shlomo ${ }^{49}$, N. Sattar ${ }^{50}$, S. E. Baumeister ${ }^{51}$, G. Davey Smith ${ }^{15}$, \\ J. P. Casas ${ }^{52,53}$, D. K. Houstonn ${ }^{54}$, W. März ${ }^{55,56,57}$, K. Christensen ${ }^{10,58,59}$, V. Gudnason ${ }^{8,9}$, F. B. Hu ${ }^{60,61}$, \\ A. Metspalu' ${ }^{6}$ P. M. Ridker ${ }^{4,5}$, N. J. Wareham³ , R. J. F. Loos ${ }^{3,62}$, H. Tiemeier ${ }^{2,63,64}$, E. Sonestedt ${ }^{65}$ and \\ T. I. A. Sørensen ${ }^{1,15,66}$
}

${ }^{1}$ Institute of Preventive Medicine, Bispebjerg and Frederiksberg Hospitals, The Capital Region, Copenhagen, Denmark; ${ }^{2}$ Department of Epidemiology, Erasmus Medical Centre, Rotterdam, The Netherlands; ${ }^{3}$ MRC Epidemiology Unit, Institute of Metabolic Science, Addenbrooke's Hospital, University of Cambridge, Cambridge, UK; ${ }^{4}$ Division of Preventive Medicine, Brigham and Women's Hospital, Boston, Massachusetts, USA; ${ }^{5} \mathrm{Harvard}$ Medical School, Boston, Massachusetts, USA; ${ }^{6}$ Estonian Genome Center, University of Tartu, Tartu, Estonia; ${ }^{7}$ Department of Epidemiology and Population

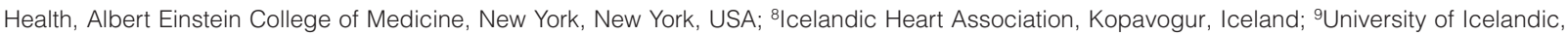
Reykajvik, Iceland; ${ }^{10}$ The Danish Aging Research Center and The Danish Twin Registry, Epidemiology, Biostatistics and Biodemography, Institute of Public Health, University of Southern Denmark, Odense, Denmark; ${ }^{11}$ Mannheim Institute of Public Health, Social and Preventive Medicine, Medical Faculty Mannheim, Heidelberg University, Mannheim, Germany; ${ }^{12}$ Laboratory of Neurogenetics, Intramural Research Program, National Institute on Aging, Bethesda, Maryland, USA; ${ }^{13}$ Department of Cardiology, Leiden University Medical Center, Leiden, The Netherlands; ${ }^{14}$ Department of Gerontology and Geriatrics, Leiden University Medical Center, Leiden, The Netherlands; ${ }^{15} \mathrm{MRC}$ Integrative Epidemiology Unit (IEU), University of Bristol, Bristol, UK; ${ }^{16}$ Institute for Medical Informatics, Biometry and Epidemiology, University of Duisburg-Essen, Essen, Germany; ${ }^{17}$ Department of Biostatistics and Center for Statistical Genetics, University of Michigan, Ann Arbor, Michigan, USA; ${ }^{18}$ Department of Clinical Chemistry, Fimlab Laboratories, Tampere, Finland; ${ }^{19}$ School of Medicine, University of Tampere, Tampere, Finland; ${ }^{20}$ Department of Cardiology, University Medical Center Groningen, University of Groningen, Groningen, The Netherlands; ${ }^{21}$ Department of Medicine I, Ludwig-Maximilians-University, Munich, Germany; ${ }^{22}$ Institute of Medical Informatics, Biometry and Epidemiology, Chair of Genetic Epidemiology, Ludwig-Maximilians-Universität, Munich, Germany; ${ }^{23}$ Institute of Genetic Epidemiology, Helmholtz Zentrum München - German Research Center for Environmental Health, Neuherberg, Germany; ${ }^{24}$ Deutsches Forschungszentrum für Herz-Kreislauferkrankungen (DZHK), Partner site Munich Heart Alliance, Munich, Germany; ${ }^{25}$ Karolinska Institutet, Institute of Environmental Medicine, Unit of Cardiovascular Epidemiology, Stockholm, Sweden; ${ }^{26}$ Department of Internal Medicine, Internal Medicine, Lausanne University Hospital (CHUV), Lausanne, Switzerland; ${ }^{27} \mathrm{MRC}$ Unit for Lifelong Health and Ageing, University College London, London, UK; ${ }^{28}$ Department of Molecular Epidemiology, German Institute of Human Nutrition Potsdam-Rehbrücke, Nuthetal, Germany; ${ }^{29}$ Department of Epidemiology, German Institute of Human Nutrition Potsdam-Rehbrücke, Nuthetal, Germany; ${ }^{30}$ QIMR Berghofer Medical Research Institute, Brisbane, Australia; ${ }^{31}$ Atherosclerosis Research Unit, Department of Medicine Solna, Karolinska Institutet, Stockholm, Sweden; ${ }^{32}$ Institute of Regional Health Services Research and Odense Patient data Explorative Network, Odense University Hospital, Odense, Denmark; ${ }^{33}$ Institute of Public Health and Clinical Nutrition, University of Eastern Finland, Kuopio, Finland; ${ }^{34}$ Danish Cancer Society Research Centre, Copenhagen, Denmark; ${ }^{35}$ Diabetes Prevention Unit, Department of Chronic Disease Prevention, National Institute for Health and Welfare, Helsinki, Finland; ${ }^{36}$ Department of General Practice and Primary Health Care, Institute of Clinical Medicine, University of Helsinki, Helsinki, Finland; ${ }^{37}$ Folkhälsan Research Centre, Helsinki, Finland; ${ }^{38}$ Unit of General Practice, Helsinki University Central Hospital, Helsinki, Finland; ${ }^{39}$ Institute of Epidemiology II, Helmholtz Zentrum München - German Research Center for Environmental Health, Neuherberg, Germany; ${ }^{40}$ Department of Genetic, University Medical Center Groningen, University of Groningen, Groningen, The Netherlands; ${ }^{41}$ Durrer Center for Cardiogenetic Research, ICIN-Neterlands Heart Institute, Utrecht, The Netherlands; ${ }^{42}$ Department of Epidemiology and Public Health, University College London, London, UK;

*These authors have contributed equally to this work. 
${ }^{43}$ ISER, University of Essex, Colchester, UK; ${ }^{44}$ Inserm, U744, Institut Pasteur de Lille, University Lille Nord de France, Lille, France; ${ }^{45}$ Diabetes Prevention Unit, National Institute for Health and Welfare, Helsinki, Finland; ${ }^{46}$ Centre for Vascular Prevention, Danube-University Krems, Krems, Austria; ${ }^{47}$ Instituto de Investigacion Sanitaria del Hospital Universario LaPaz (IdiPAZ), Madrid, Spain; ${ }^{48}$ Diabetes Research Group, King Abdulaziz University, Jeddah, Saudi Arabia; ${ }^{49}$ School of Social and Community Medicine, University of Bristol, Bristol, UK; ${ }^{50} \mathrm{BHF}$ Glasgow Cardiovascular Research Centre, Faculty of Medicine, Glasgow, UK; ${ }^{51}$ Institute for Community Medicine, University Medicine Greifswald, Greifswald, Germany; ${ }^{52}$ Department of Non-Communicable Disease Epidemiology, London School of Hygiene and Tropical Medicine, London, UK; ${ }^{53}$ Institute of Cardiovascular Science, University College London, London, UK; ${ }^{54}$ Department of Internal Medicine, Section on Gerontology and Geriatric Medicine, Wake Forest School of Medicine, Winston Salem, North Carolina, USA; ${ }^{55}$ Vth Department of Medicine (Nephrology, Hypertensiology, Endocrinology, Diabetology, Rheumatology), Medical Faculty of Mannheim, University of Heidelberg, Mannheim, Germany; ${ }^{56}$ Clinical Institute of Medical and Chemical Laboratory Diagnostics, Medical University of Graz, Graz, Austria; ${ }^{57}$ Synlab Academy, Synlab Services GmbH, Mannheim, Germany; ${ }^{58}$ Department of Clinical Genetics, Odense University Hospital, Odense, Denmark; ${ }^{59}$ Department of Clinical Biochemistry and Pharmacology, Odense University Hospital, Odense, Denmark; ${ }^{60}$ Department of Nutrition, Harvard School of Public Health, Boston, Massachusetts, USA; ${ }^{61}$ Department of Epidemiology, Harvard School of Public Health, Boston, Massachusetts, USA; ${ }^{62}$ The Charles Bronfman Institute for Personalized Medicine, The Mindich Child Health and Development Institute, The Genetics of Obesity and Related Metabolic Traits Program, Icahn School of Medicine at Mount Sinai, New York, New York, USA; ${ }^{63}$ Department of Child and Adolescent Psychiatry, Erasmus Medical Centre, Rotterdam, The Netherlands; ${ }^{64}$ Department of Psychiatry, Erasmus Medical Centre, Rotterdam, The Netherlands; ${ }^{65}$ Department of Clinical Sciences Malmö, Lund University, Malmö, Sweden; ${ }^{66}$ Novo Nordisk Foundation Centre for Basic Metabolic Research, Section on Metabolic Genetics, Faculty of Health and Medical Sciences, University of Copenhagen, Frederiksberg, Denmark

TThe FTO-Mortality Collaborating Group, contributing cohort data, includes the following:

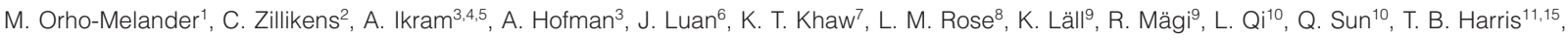
L. J. Launer ${ }^{11}$, G. Eiriksdottir ${ }^{12}$, M. E. Kleber ${ }^{13}$, G. Delgado ${ }^{13}$, Y. Liu' ${ }^{14}$, M. Garcia ${ }^{15}$, A. Teumer ${ }^{16}$, H. Grabe ${ }^{17}$, G. Homuth ${ }^{18}$, J. W. Jukema ${ }^{19,20,21}$,

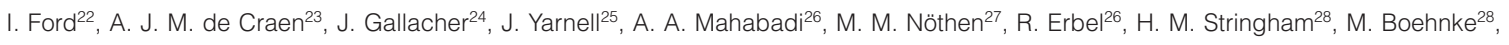
P. Amouyel ${ }^{29}$, J. Ferrières ${ }^{30,31,32}$, D. Arveiler ${ }^{33}$, M. Kähönen ${ }^{34,35}$, K. Nikus ${ }^{36}$, T. Nieminen ${ }^{37,38,39}$, A. Sanchez ${ }^{40}$, M. Kivimaki ${ }^{40}$, J. V. van Vliet-Ostaptchouk ${ }^{41,42}$, R. Hampel ${ }^{43}$, B. Thorand ${ }^{43}$, U. De Faire ${ }^{44}$, F. Nyberg ${ }^{45,46}$, D. Kuh K $^{47}$, N. G. Martin ${ }^{48}$, G. W. Montgomery ${ }^{48}$, A. C. Heath ${ }^{49}$, P. A. F. Madden ${ }^{49}$, C. Osmond ${ }^{50}$, N. Pulizzi ${ }^{51}$, N. Roswall ${ }^{52}$, J. Halkjær ${ }^{52}$, K. Overvad ${ }^{53,54}$, M. Uusitupa ${ }^{55,56}$, L. Kinnunen ${ }^{57}$, J. Lindström ${ }^{57}$, J. Saramies ${ }^{58}$, S. Keinänen-Kiukaanniemi59, H. Uusitalo60, E. Hussi ${ }^{58}$, D. Baldassarre ${ }^{61,62}$, F. Veglia ${ }^{62}$, S. Humphries ${ }^{63}$, E. Tremoli61,62 and B. Heitmann ${ }^{64}$ ${ }^{1}$ Department of Clinical Sciences Malmö, Lund University, Malmö, Sweden; ${ }^{2}$ Department of Internal Medicine, Erasmus Medical Centre, Rotterdam, The Netherlands; ${ }^{3}$ Department of Epidemiology, Erasmus Medical Centre, Rotterdam, The Netherlands; ${ }^{4}$ Department of Neurology, Erasmus Medical Centre, Rotterdam, The Netherlands; ${ }^{5}$ Department of Radiology, Erasmus Medical Centre, Rotterdam, The Netherlands; ${ }^{6}$ MRC Epidemiology Unit, Institute of Metabolic Science, Addenbrooke's Hospital, University of Cambridge, Cambridge, UK; ${ }^{7} \mathrm{MRC}$ Centre for Nutritional Epidemiology in Cancer Prevention and Survival, Department of Public Health and Primary Care, University of Cambridge, Cambridge, UK; ${ }^{8}$ Division of Preventive Medicine, Brigham and Women's Hospital, Boston, Massachusetts, USA; ${ }^{9}$ Estonian Genome Center, University of Tartu, Tartu, Estonia; ${ }^{10}$ Department of Nutrition, Harvard School of Public Health, Boston, Massachusetts, USA; ${ }^{11}$ Laboratory of Epidemiology, Demography, and Biometry, National Institute on Aging, National Institutes of Health, Bethesda, Maryland, USA; ${ }^{12}$ Icelandic Heart Association, Kopavogur, Iceland; ${ }^{13}$ Vth Department of Medicine (Nephrology, Hypertensiology, Endocrinology, Diabetology, Rheumatology), Medical Faculty of Mannheim, University of Heidelberg, Mannheim, Germany; ${ }^{14}$ Department of Epidemiology and Prevention, Division of Public Health Sciences, Wake Forest School of Medicine, Winston Salem, North Carolina, USA; ${ }^{15}$ Laboratory of Epidemiology and Population Sciences, Intramural Research Program, National Institute on Aging, Bethesda, Maryland, USA; ${ }^{16}$ Institute for Community Medicine, University Medicine Greifswald, Greifswald, Germany; ${ }^{17}$ Department of Psychiatry and Psychotherapy, University Medicine Greifswald, Greifswald, Germany; ${ }^{18}$ Interfaculty Institute for Genetics and Functional Genomics, University Medicine and Ernst-MoritzArndt-University Greifswald, Greifswald, Germany; ${ }^{19}$ Department of Cardiology, Leiden University Medical Center, Leiden, The Netherlands; ${ }^{20}$ Durrer Center for Cardiogenetic Research, Amsterdam, The Netherlands; ${ }^{21}$ Interuniversity Cardiology Institute of the Netherlands, Utrecht, The Netherlands; ${ }^{22}$ Robertson Center for Biostatistics, University of Glasgow, Glasgow, UK; ${ }^{23}$ Department of Gerontology and Geriatrics, Leiden University Medical Center, Leiden, The Netherlands; ${ }^{24}$ Institute of Primary Care and Public Health, Cardiff University School of Medicine, Cardiff, UK; ${ }^{25}$ Epidemiology Research Group, Queen's University Belfast, Belfast, UK; ${ }^{26}$ West-German Heart Center Essen, Department of Cardiology, University of Duisburg-Essen, Essen, Germany; ${ }^{27}$ Institute of Human Genetics, University of Bonn, Bonn, Germany; ${ }^{28}$ Department of Biostatistics and Center for Statistical Genetics, University of Michigan, Ann Arbor, Michigan, USA; ${ }^{29}$ Inserm, U744, Institut Pasteur de Lille, University Lille Nord de France, UDSL, Lille, France; ${ }^{30}$ Hôpital Rangueil, Toulouse, France; ${ }^{31}$ INSERM U1027, Toulouse, France; ${ }^{32}$ Université Paul Sabatier Toulouse, Toulouse, France; ${ }^{33}$ Department of Epidemiology and Public Health, University of Strasbourg, Strasbourg, France; ${ }^{34}$ Department of Clinical Physiology, Tampere University Hospital, Tampere, Finland; ${ }^{35}$ School of Medicine, University of Tampere, Tampere, Finland; ${ }^{36}$ Heart Hospital Co., Department of Cardio-Thoracic Surgery, Tampere University Hospital, Tampere, Finland; ${ }^{37}$ Division of Cardiology, Helsinki University Central Hospital, Helsinki, Finland; ${ }^{38}$ Department of Internal Medicine, Päijät-Häme Central Hospital, Lahti, Finland; ${ }^{39}$ Department of Clinical Pharmacology, University of Tampere, Tampere, Finland; ${ }^{40}$ Department of Epidemiology and Public Health, University College London, London, UK; ${ }^{41}$ Department of Endocrinology, University Medical Center Groningen, University of Groningen, Groningen, The Netherlands; ${ }^{2}$ Unit of Genetic Epidemiology and 
Received 3 December 2014; accepted 12 December 2014

Address for correspondence: $\operatorname{Dr} \mathrm{E}$ Zimmermann, Institute of Preventive Medicine, Frederiksberg Hospital, Nordre Fasanvej 57, Hovedvejen, Indgang 5, stuen, 2000 Frederiksberg, Denmark.

E-mail: esther.zimmermann@regionh.dk

\section{Summary}

Previously, a single nucleotide polymorphism (SNP), rs9939609, in the FTO gene showed a much stronger association with all-cause mortality than expected from its association with body mass index (BMI), body fat mass index (FMI) and waist circumference (WC). This finding implies that the SNP has strong pleiotropic effects on adiposity and adiposity-independent pathological pathways that leads to increased mortality. To investigate this further, we conducted a meta-analysis of similar data from 34 longitudinal studies including 169,551 adult Caucasians among whom 27,100 died during follow-up. Linear regression showed that the minor allele of the FTO SNP was associated with greater BMI $(n=169,551$; $0.32 \mathrm{~kg} \mathrm{~m}^{-2} ; 95 \%$ CI $\left.0.28-0.32, P<1 \times 10^{-32}\right)$, WC $(n=152,631 ; 0.76 \mathrm{~cm}$; $\left.0.68-0.84, \quad P<1 \times 10^{-32}\right)$ and FMI $\left(n=48,192 ; \quad 0.17 \mathrm{~kg} \mathrm{~m}^{-2} ; \quad 0.13-0.22\right.$, $\left.P=1.0 \times 10^{-13}\right)$. Cox proportional hazard regression analyses for mortality showed that the hazards ratio (HR) for the minor allele of the FTO SNPs was 1.02 (1.00-1.04, $P=0.097)$, but the apparent excess risk was eliminated after adjustment for BMI and WC (HR: 1.00; 0.98-1.03, $P=0.662$ ) and for FMI (HR: 1.00; $0.96-1.04, P=0.932)$. In conclusion, this study does not support that the FTO SNP is associated with all-cause mortality independently of the adiposity phenotypes.

Keywords: FTO, meta-analysis, mortality, obesity.

obesity reviews (2015) 16, 327-340

\section{Introduction}

In 2007, genome-wide association studies discovered the first obesity susceptibility locus, the 'fat mass and obesity associated gene' (FTO) (1,2). A cluster of common single nucleotide polymorphisms (SNPs) in the first intron of FTO was identified as those carrying the association. Each additional minor A-allele of the rs9939609 SNP in the FTO cluster is associated with increased body mass index (BMI) by $\sim 0.40 \mathrm{~kg} \mathrm{~m}^{-2}$ (1) and an increased risk of obesity by
$20-30 \%(1,3)$. This FTO SNP appears to influence primarily the size of the overall fat mass irrespective of the body fat distribution (4). Thus, it is expected that the FTO SNP would also be associated with the various healthdamaging effects of adiposity. Indeed, several analyses using the FTO SNP as instrumental variable of adiposity confirmed the causality of the association between adiposity and its detrimental health effects (5-7).

It would therefore be expected that the FTO SNP is also associated with all-cause mortality. In observational

The FTO-Mortality Collaborating Group, contributing cohort data, continued:

Bioinformatics, Department of Epidemiology, University Medical Center Groningen, University of Groningen, Groningen, The Netherlands; ${ }^{43}$ Institute of Epidemiology II, Helmholtz Zentrum München - German Research Center for Environmental Health, Neuherberg, Germany; ${ }^{44}$ Division of Cardiovascular Epidemiology, Institute of Environmental Medicine, Karolinska Institutet, Stockholm, Sweden; ${ }^{45}$ Occupational and Environmental Medicine, Department of Public Health and Community Medicine, Sahlgrenska Academy, University of Gothenburg, Gothenburg, Sweden; ${ }^{46}$ AstraZeneca R\&D, Mölndal, Sweden; ${ }^{47} \mathrm{MRC}$ Unit for Lifelong Health and Ageing, University College London, London, UK; ${ }^{48} \mathrm{QIMR}$ Berghofer Medical Research Institute, Brisbane, Queensland, Australia; ${ }^{49}$ Department of Psychiatry, Washington University, St Louis, Missouri, USA; ${ }^{50}$ MRC Epidemiology Resource Centre, Southampton General Hospital, University of Southampton, Southampton, UK; ${ }^{51}$ Department of Endocrinology and Metabolism, University of Pisa, Pisa, Italy; ${ }^{52}$ Danish Cancer Society Research Centre, Copenhagen, Denmark; ${ }^{53}$ Department of Cardiology, Aalborg University Hospital, Aalborg, Denmark; ${ }^{54}$ Department of Public Health, Section for Epidemiology, Aarhus University, Aarhus, Denmark; ${ }^{55}$ Institute of Public Health and Clinical Nutrition, University of Eastern Finland, Kuopio, Finland; ${ }^{56}$ Research Unit, Kuopio University Hospital, Kuopio, Finland; ${ }^{57}$ Diabetes Prevention Unit, National Institute for Health and Welfare, Helsinki, Finland; ${ }^{58}$ South Karelia Central Hospital, Lappeenranta, Finland; ${ }^{59}$ Faculty of Medicine, Institute of Health Sciences, Oulu University, Oulu, Finland; ${ }^{60}$ Department of Ophthalmology, Tampere University, Tampere, Finland; ${ }^{61}$ Dipartimento di Scienze Farmacologiche e Biomolecolari, Università di Milano, Milan, Italy; ${ }^{62}$ Centro Cardiologico Monzino, IRCCS, Milan, Italy; ${ }^{63}$ Centre for Cardiovascular Genetics, University College London, London, UK; ${ }^{64}$ Institute of Preventive Medicine, Bispebjerg and Frederiksberg Hospitals, The Capital Region, Copenhagen, Denmark 
studies, an underestimation of the strength of the association between adiposity and mortality is suspected because of the likely confounding and reverse causality, as indicated in a previous study using the BMI of children as instrumental variable for BMI of the parents (8). However, a recent study indicated that the FTO rs9939609 may have a much stronger association with all-cause mortality than could plausibly be attributed to such biases (9). While the SNP showed the expected association with BMI, it was also associated with a doubling of mortality (dominant genetic model), even after adjusting for waist circumference (WC), fat mass index (FMI = body fat mass/height ${ }^{2} ; \mathrm{kg} \mathrm{m}^{-2}$ ) and BMI at younger ages. No distinct associations were found with any of the major causes of death or preceding disease incidence that could explain the finding.

On this basis, we speculate that the FTO SNP or some other SNPs tightly linked to it in the genomic region may have a major pleiotropic effect influencing pathways implicated in the disease processes leading to increased risk of dying independent of body weight regulation. To test the hypothesis that the FTO SNP rs9939609 (or any proxy SNP, $r^{2}>0.80$ ) was associated with all-cause mortality, with and without adjustment for BMI, WC and FMI, we conducted a meta-analysis based on individual data of Caucasian men and women from multiple studies.

\section{Populations and methods}

\section{Study selection}

We planned a meta-analysis based on novel analyses of longitudinal data. We performed a PubMed search using the search terms 'FTO and obesity' to identify potential contributing studies. Further studies were identified via the network of collaborators who joined the meta-analysis and through e-mail within the GIANT (www.broadinstitute .org/collaboration/giant/index.php/GIANT_consortium)

and CHARGE (web.chargeconsortium.com) consortia. All studies that included Caucasian populations and that were able to provide baseline data on BMI and the FTO SNP genotype, as well as mortality data during the following observation time, were considered as eligible (Fig. 1). This resulted in a total of 34 studies (Table S1) (10-42). They included 37 cohorts, as in three studies data were analysed separately for cohorts established on the basis of follow-up of previously formed cases and controls (Health Professionals Follow-up Study (HPFS) (26), Nurses' Health Studies (NHS) (32) and Stockholm Heart Epidemiology program (SHEEP) (39)).

\section{Genotyping}

If available, genotyping of the rs9939609 SNP was preferred, but a proxy SNP in high linkage disequilibrium with the rs9939609 was used instead in the following studies:

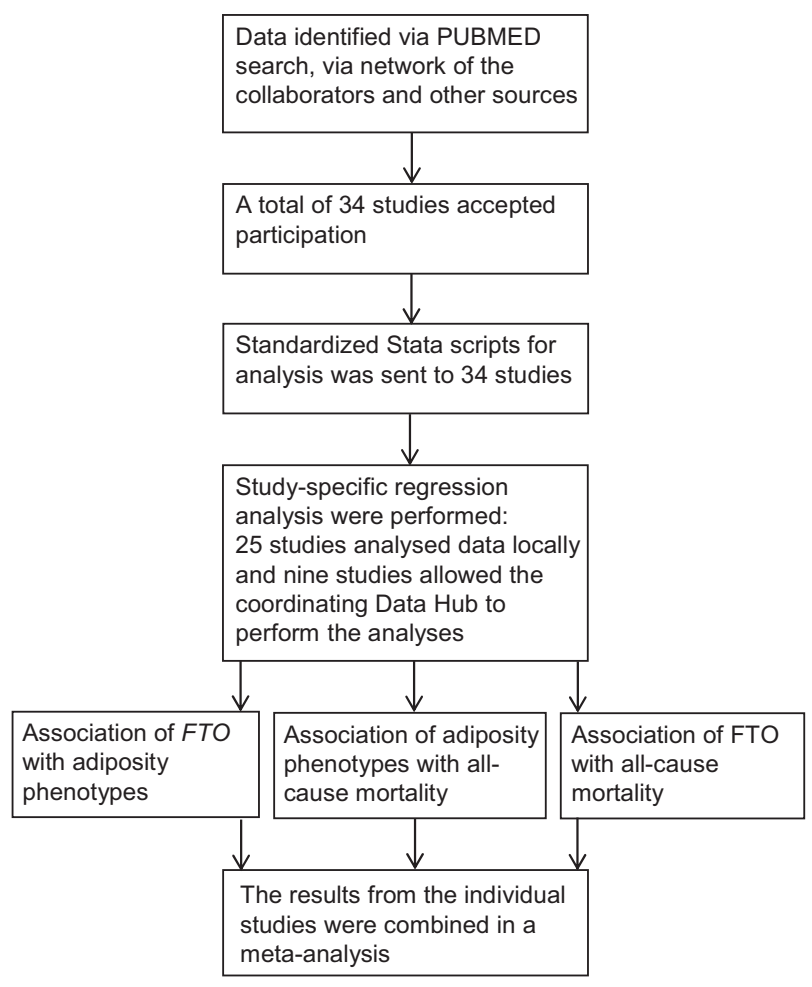

Figure 1 Study design of the FTO-mortality meta-analysis.

Eligible studies were identified by a literature search, as well as through network of the collaborators and calls in the international consortia GIANT and CHARGE (labelled 'other sources' in the figure). Of all studies that were invited, 34 studies of adults $(n=169,551)$ participated in the meta-analysis. Standardized Stata scripts were sent to each of the studies, 25 studies analysed data locally and nine studies were analysed centrally at the coordinating Data Hub. All the local estimates were meta-analysed.

EPIC Norfolk (rs1121980; $\left.\mathrm{r}^{2}=0.84\right)$ (18), EPIC-Potsdam (rs9935401; $\left.\mathrm{r}^{2}=0.98\right)$ (19) and Heinz Nixdorf Recall Study (rs8050136; $\mathrm{r}^{2}=1$ ) (25). In The Health ABC study (http://www.grc.nia.nih.gov/branches/leps/healthabc/), the genotype was generated by 1,000 genome imputation. Only genotype data that met the local quality control criteria, i.e. genotyping call rate, concordance in duplicate samples and tests for deviations from Hardy-Weinberg equilibrium, were used in the analyses.

\section{Measurement of adiposity phenotype}

BMI was calculated for each individual on the basis of measured height and weight (exceptions were the use of self-reported data, which have been validated, from The Danish 1905-Cohort (15), the Danish Diet, Cancer, and Health Cohort (DCH) (16), the NHS (32) and the HPFS (26)). Furthermore, 32 of the 37 cohorts included data on WC $(10,12,14,16-35,37-42)$, which were measured at the same time as height and weight, and 10 studies had information on total body fat mass measured by bio-impedance 
from which FMI was calculated $(10,14,19,21,23$, $24,29,30,42)$.

\section{Study-specific analyses}

All the study-specific analyses were planned to be performed by local analysts according to a centrally prepared analysis plan for the covariates and through usage of a hierarchical set of Stata scripts (Stata 12.1; StataCorp LP, College Station, TX, USA; 2012), developed by the Data Hub at the Institute of Preventive Medicine, Copenhagen, Denmark. The local analysts were asked to check the accuracy of their variable assignments and additional checks were later performed at the Data Hub. Any detected ambiguities were clarified with the respective study investigators before the final meta-analysis stage. All the derived effect estimates of the local analyses along with their standard errors were automatically saved in corresponding Stata datasets that were returned to the Data Hub. Alternatively, data were sent directly and analysed using the same scripts by the Data Hub analyst (LHÄ), which was performed for nine studies $(12,16,21,23,24,30,31,40,42)$.

For the main analyses, the effect of the FTO SNP was tested assuming an additive allelic effect. However, because in the previous study the effect of the FTO SNP on mortality fitted a dominant genetic model (9), this model was also investigated. In order to take into account the variation in sex and age, all adiposity phenotypes were subsequently analysed adjusting for sex and age using residuals from regressions of the phenotypes on sex, age, age-squared, age $\times$ sex or age-squared $\times$ sex.

Each study tested the association between the FTO genotype and the adiposity phenotypes using linear regression. Further, each study tested for a main effect on mortality of FTO genotype, BMI, WC and FMI, respectively. In addition, the associations of the combined adiposity phenotypes, (BMI and WC) and (FMI and WC), with mortality were estimated. Finally, the association between the FTO genotype and mortality was examined adjusting for the single and combined adiposity phenotypes.

Due to the distinct U-shaped association between BMI and mortality $(43,44)$, where the increased mortality may not reflect associations with fat mass (45), individuals with $\mathrm{BMI}<20 \mathrm{~kg} \mathrm{~m}^{-2}$ were excluded from the analyses. However, as the nadir of the $\mathrm{U}$ curve has often been reported to be around $25 \mathrm{~kg} \mathrm{~m}^{-2}(43,44)$, there may be a $\mathrm{J}$-shaped relationship between BMI above $20 \mathrm{~kg} \mathrm{~m}^{-2}$ and mortality. Similarly, the association between WC and mortality has been reported to be U-shaped (46-48). Studies have shown that WC adjusted for BMI and BMI adjusted for WC have monotonic associations with all-cause mortality in opposite directions; positive and linear for the WC and decreasing and flattening out for the BMI $(46,48)$. Therefore, in the corresponding regression models, both
BMI and WC were simultaneously included as linear, quantitative trait variables. FMI has a linear relationship with mortality $(45,49)$ and was hence included as a quantitative trait variable in separate analyses. In additional analyses, it was also adjusted for WC, which appears to capture the effect of the FMI on mortality (49).

The Cox proportional hazards regression model was used for the analyses of mortality and the outcome is expressed as hazard ratios (HR) per unit difference of the covariates. The participants were followed from date of blood collection for DNA (or from the date of anthropometric measurements if that took place later) until death, censoring because of loss to follow-up, emigration or end of follow-up. To ensure optimal adjustment for age as a major determinant of mortality, we used age as the underlying time scale in the Cox regression. This implied entrance of the individual into the estimation of the HR at the age when the follow-up began and hence from the age at which the individual was considered at risk of dying. Moreover, as the possible pleiotropic effect might be most distinct in the older segments of the cohorts (because of the higher mortality), we also performed subgroup analyses restricting the minimum age-at-risk to 60, 65 and 70 years, respectively. All Cox regression analyses were adjusted for sex and lack of difference in association between the two sexes was confirmed. The proportional hazard assumptions were assessed by a test based on Schoenfeld residuals (50) using the Stata estat phtest command; only one of 37 studies (EPIC-Potsdam) showed a significant deviation $(P=0.01)$.

\section{Meta-analysis}

The locally estimated regression coefficients and standard errors were combined by inverse variance weighted metaanalyses with random effects $(51,52)$, using the Stata metan command (53). In all the meta-analyses, between-study heterogeneity was tested for by the $\mathrm{Q}$ statistic and quantified by the $\mathrm{I}^{2}$ value; $\mathrm{I}^{2}$ values of $<25 \%, 25-75 \%$ and $>75 \%$ were defined as low, moderate and high heterogeneity, respectively $(53,54)$. If the heterogeneity turned out to be moderate or high, meta-regression analysis was planned in order to search for and adjust for the sources of heterogeneity.

\section{Results}

\section{Study characteristics}

The 37 participating cohorts provided data on 70,020 men of whom 13,857 died during follow-up and 99,531 women of whom 13,243 died during follow-up (Table S1). Regarding the mean follow-up time over cohorts, the median length was 9.4 years (range of means 2.9-20.0 years). Mean baseline age ranged from 38 to 93 years. The minor allele frequency of the FTO SNP ranged from $34 \%$ to $45 \%$. 
Table 1 Association of the minor (A) allele of the rs9939609 SNP in FTO with BMI, WC and FMI, respectively, in a random effects meta-analysis of Caucasian adults

\begin{tabular}{lrll}
\hline Phenotype & $n$ & $\beta(95 \% \mathrm{Cl})$ & $P$-value \\
\hline BMI $\left(\mathrm{kg} \mathrm{m}^{-2}\right)$ & 169,551 & $0.32(0.28-0.32)$ & $<1 \times 10^{-32}$ \\
WC $\left(\mathrm{cm}^{-3}\right.$ & 152,631 & $0.76(0.68-0.84)$ & $<1 \times 10^{-32}$ \\
FMl $\left(\mathrm{kg} \mathrm{m}^{-2}\right)$ & 48,192 & $0.17(0.13-0.22)$ & $1.0 \times 10^{-13}$
\end{tabular}

$\beta$, estimated difference in phenotype per minor allele of the rs9939609 or a proxy $\left(r^{2}>0.8\right)$; BMl, body mass index; $\mathrm{Cl}$, confidence interval; FMI, fat mass index; $n$, number of individuals; SNP, single nucleotide polymorphism; WC, waist circumference.

\section{Association of FTO with adiposity phenotypes}

The association between the FTO SNP and BMI and WC was confirmed; each additional minor allele of the FTO SNP was associated with a $0.32 \mathrm{~kg} \mathrm{~m}^{-2}$ higher BMI and with a $0.76 \mathrm{~cm}$ higher WC (Table 1 ). In the 10 cohorts with data on FMI, each FTO minor allele was associated with a $0.17 \mathrm{~kg} \mathrm{~m}^{-2}$ higher FMI (Table 1).

\section{Association of adiposity phenotypes with all-cause mortality}

All-cause mortality was positively associated with BMI (HR per unit BMI of 1.02, 95\% confidence interval [CI]: $\left.1.01-1.03 ; P=1.0 \times 10^{-8}\right)$, but the association went in the opposite direction when BMI was concurrently adjusted for WC (HR: $0.97,95 \%$ CI: $0.96-0.98 ; \quad P=4.5 \times 10^{-6}$ ) (Table 2). The mortality was positively associated with WC (HR: 1.01, 95\% CI: 1.01-1.02; $P=5.6 \times 10^{-24}$ ), also when concurrently adjusted for BMI (HR: 1.02, 95\% CI: $1.02-$ 1.03; $P=8.8 \times 10^{-18}$ ) (Table 2 ). Mortality was positively associated with FMI alone (HR: 1.05 (95\% CI: 1.02-1.08; $P=0.003)$, but negatively associated when adjusted for WC (HR: 0.97, 95\% CI: 0.95-0.99; $P=0.009$ ) (Table 2).

\section{Associations of FTO with all-cause mortality}

FTO was associated (albeit non-significantly) with all-cause mortality with a HR of 1.02 (95\% CI: 1.00-1.04; P = 0.097) per minor allele. Adjustment for BMI and WC attenuated the estimate to a HR of 1.00 (95\% CI: 0.98 1.03; $P=0.662$ ) (Fig. 3).

In the subset of 10 studies including 48,192 individuals with FMI data (6,436 deaths), each minor FTO allele was associated with mortality with a HR of 1.01 (95\% CI: $0.96-1.06 ; P=0.731)$ and a HR of 1.00 (95\% CI: $0.96-$ 1.04; $P=0.932$ ) when adjusted for FMI (Fig. 4).

The analyses assuming a dominant genetic model showed no association between FTO and mortality (HR of 1.00, 95\% CI: 0.98-1.03; $P=0.901)$. The age-restricted
Table 2 Association of BMI, WC and FMI with all-cause mortality in a random effects meta-analysis of Caucasian adults

\begin{tabular}{lrrll}
\hline Phenotype & $n$ & Deaths & HR $(95 \% \mathrm{Cl})$ & $P$-value \\
\hline & & & & \\
BMI $\left(\mathrm{kg} \mathrm{m}^{-2}\right)^{\star}$ & 169,551 & 27,100 & $1.02(1.01-1.03)$ & $1.0 \times 10^{-8}$ \\
BMI $\left(\mathrm{kg} \mathrm{m}^{-2}\right)$ IWC & 152,631 & 22,506 & $0.97(0.96-0.98)$ & $4.5 \times 10^{-6}$ \\
WC $(\mathrm{cm})^{\dagger}$ & 152,631 & 22,506 & $1.01(1.01-1.02)$ & $5.6 \times 10^{-24}$ \\
WC $(\mathrm{cm})$ IBMI & 152,631 & 22,506 & $1.02(1.02-1.03)$ & $8.8 \times 10^{-18}$ \\
FMI $\left(\mathrm{kg} \mathrm{m}^{-2}\right)$ & 48,192 & 6,436 & $1.05(1.02-1.08)$ & 0.003 \\
FMI $\left(\mathrm{kg} \mathrm{m}^{-2}\right)$ IWC & 48,167 & 6,433 & $0.97(0.95-0.99)$ & 0.009
\end{tabular}

BMI, body mass index; $\mathrm{Cl}$, confidence interval; FMI, fat mass index; $H R$, estimated hazard ratio of all-cause mortality per unit of the phenotype; $n$, number of individuals; WC, waist circumference; I, means adjusted for; e.g. BMI $\left(\mathrm{kg} \mathrm{m}^{-2}\right)$ IWC is BMI adjusted for waist circumference.

*Due to the assumption of linearity of the BMI-mortality association for $\mathrm{BMI} \geq 20 \mathrm{~kg} \mathrm{~m}^{-2}$, the association between high levels of BMl and mortality is potentially underestimated.

${ }^{\dagger}$ Due to the assumption of linearity of the WC-mortality association, the association between high levels of WC and mortality is potentially underestimated.

subgroup analyses (observations left-truncated with delayed entry at 60,65 and 70 years, respectively) and the sex-specific analyses showed results consistent with the non-censored analyses (Tables S2 \& S3).

The heterogeneity in all meta-analyses was very low, so there was no reason to conduct a comprehensive metaregression analysis. The possible modification of the FTOmortality association by mean age at events in each cohort was addressed by a meta-regression analysis, which did not show a significant relationship.

\section{Discussion}

In this meta-analysis, combining data of up to 169,551 adults of whom 27,100 died during follow-up, we found a very modest and statistically insignificant effect of the adiposity-associated FTO SNPs on all-cause mortality. When assuming an additive genetic effect, each minor allele increased mortality by $\sim 2 \%$ with CIs ranging from $\sim 0 \%$ to $\sim 4 \%$ (Fig. 2). When adjusting for the adiposity phenotypes, there was virtually no association between the FTO SNPs and all-cause mortality.

Our results do not support the findings from the previous study, which reported a statistically significant very strong positive association between the FTO rs9939609 SNP and all-cause mortality (9). In the previous study, FTO minor allele carriers had almost twice the mortality rate of the homozygous carriers of the major allele when analysed in a dominant genetic effect model (9). The results from the present meta-analysis provide evidence that if FTO has an effect on mortality that is not attributable to its association with the adiposity phenotypes, it is - almost without doubt 


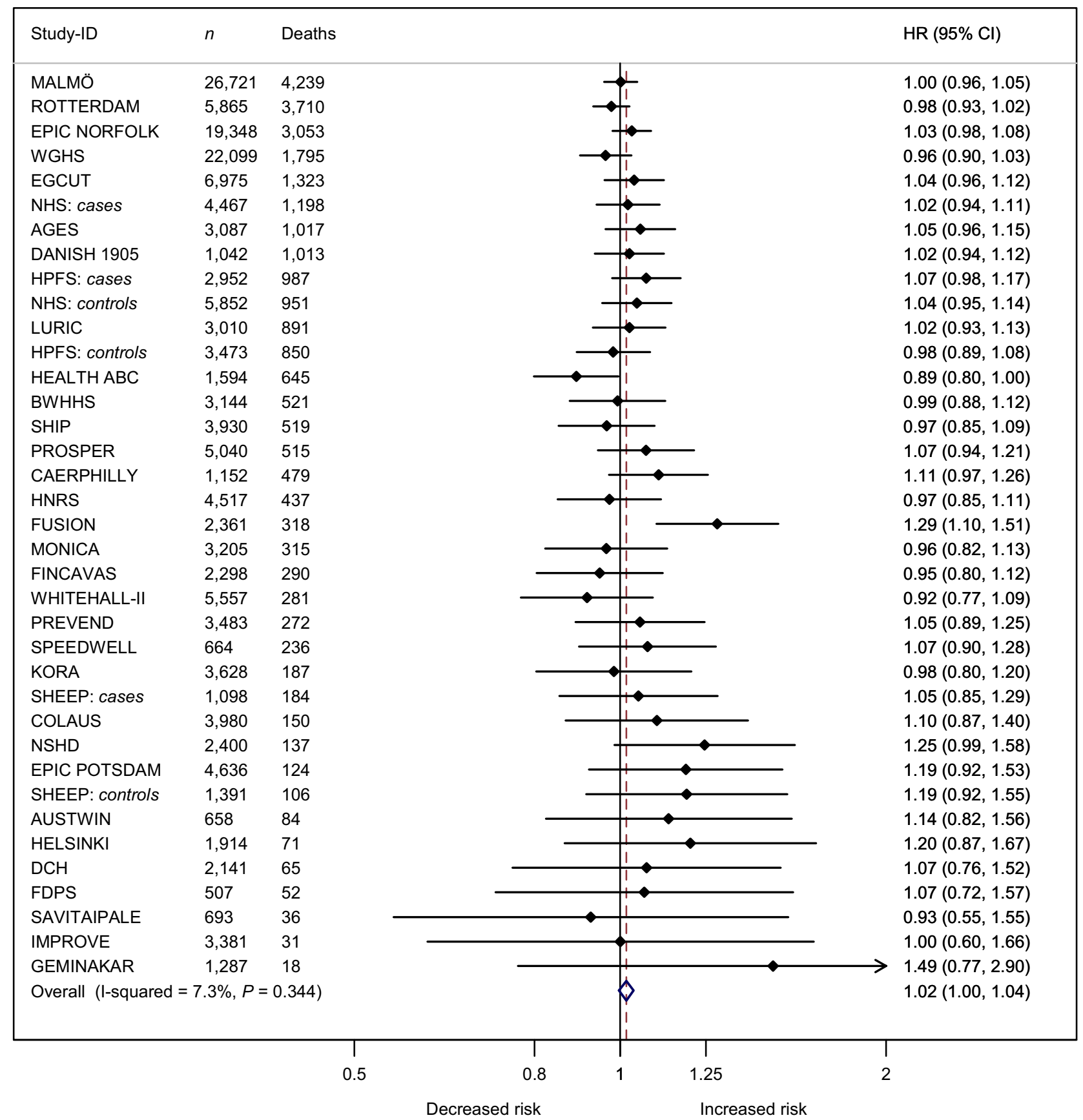

Figure 2 Forest plot of the effect of FTO rs9939609 on all-cause mortality in a random effects meta-analysis of 169,551 Caucasian adults sorted by number of deaths. The studies are sorted by decreasing sample size (the largest at the top). Details of the studies are given in Table S1. The overall estimate equalled a HR of $1.016(0.997-1.035), P=0.097$.

$95 \% \mathrm{Cl}, 95 \%$ confidence intervals; Deaths, number of deaths; HR, estimated hazard ratio of all-cause mortality per minor allele of the rs9939609 or a proxy $\left(r^{2}>0.8\right) ; n$, number of individuals.

- much smaller than that found in the previous study (as indicated by the CI). Hence, the results of the previous study are likely to be spurious, possibly reflecting random sampling errors irrespective of its statistical strength and otherwise consistent associations between the FTO SNP and the adiposity phenotypes and their associations with all-cause mortality $(4,9)$. On the other hand, several previous studies have investigated the association between FTO and cardiovascular disease and the results were recently pooled in a meta-analysis (55). The overall conclusion was that $F T O$ was associated with an increased cardiovascular risk independent of its association with BMI (55). Whether 


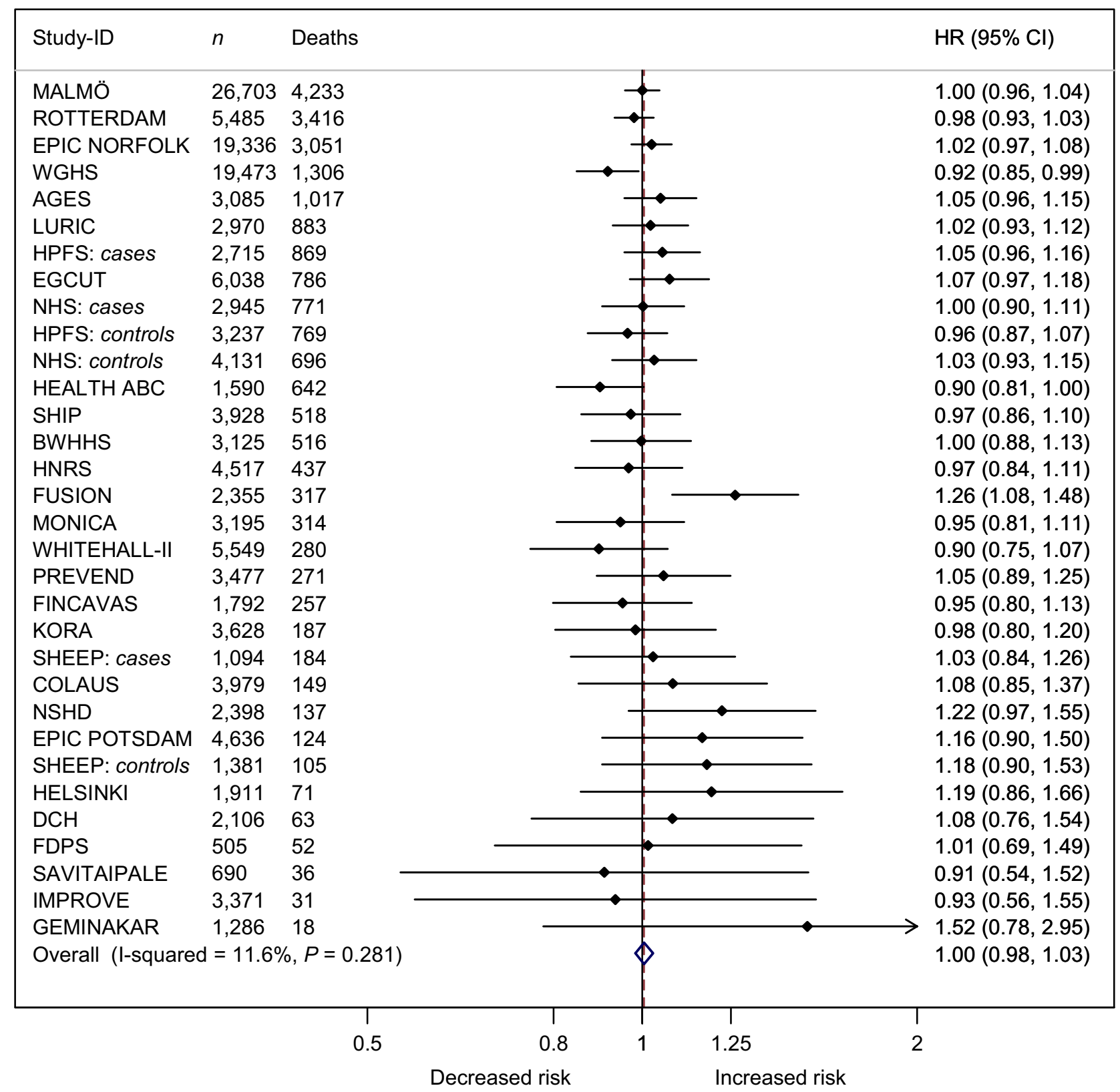

Figure 3 Forest plot of the effect of FTO rs9939609 on all-cause mortality adjusted for body mass index and waist circumference in a random effects meta-analysis of 152,631 Caucasian adults sorted by number of deaths. The studies are sorted by decreasing sample size (the largest at the top). Details of the studies are given in Table S1. The overall estimate equalled a HR 1.004 (0.983-1.027), $P=0.662$. $95 \% \mathrm{Cl}, 95 \%$ confidence intervals; Deaths, number of deaths; HR, estimated hazard ratio of all-cause mortality per minor allele of the rs9939609 or a proxy $\left(r^{2}>0.8\right) ; n$, number of individuals.

FTO has adiposity-independent effects on mortality from specific causes remains an important challenge for future research to elucidate.

The association between the FTO SNP and mortality was robust as judged from the narrow CIs and the low heterogeneity. Consistent results were also found when restricting the time-at-risk to older ages (above 60, 65 and 70 years, respectively) and to each sex, indicating no effect modifications by age and sex. When assuming a dominant effect for the minor allele, no association was found between the FTO SNP and mortality. Moreover, as addressed in the following, the associations between the FTO SNP and the adiposity phenotypes as well as the associations between the adiposity phenotypes and allcause mortality were generally as expected from previous studies. 


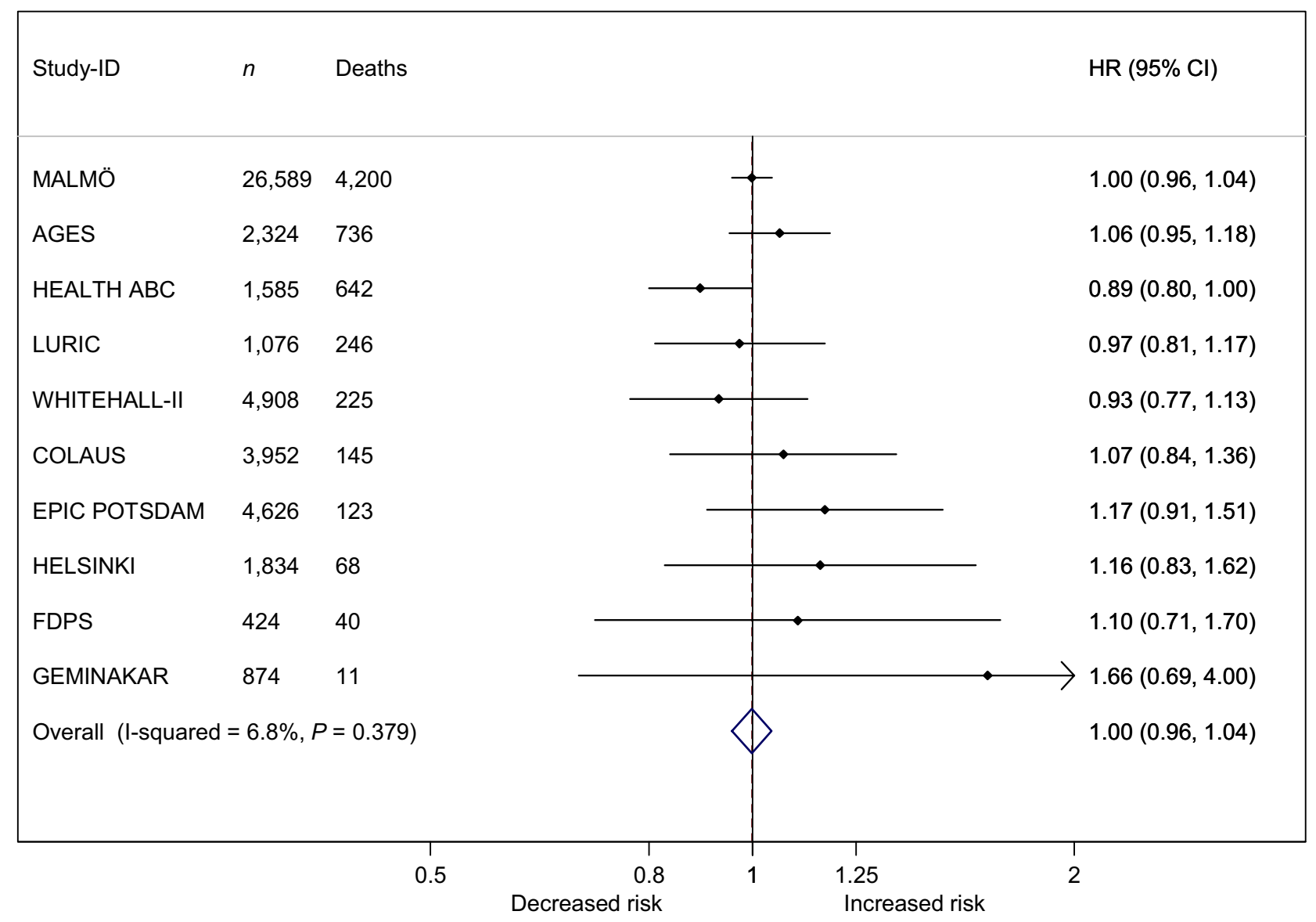

Figure 4 Forest plot of the effect of FTO rs9939609 on all-cause mortality adjusted for fat mass index in a random effects meta-analysis of 48,192 Caucasian adults sorted by number of deaths. The studies are sorted by decreasing sample size (the largest at the top). Details of the studies are given in Table S1. The overall estimate equalled a HR $0.998(0.956-1.042), P=0.932$.

$95 \% \mathrm{Cl}, 95 \%$ confidence intervals; Deaths, number of deaths; HR, estimated hazard ratio of all-cause mortality per minor allele of the rs9939609 or a proxy $\left(r^{2}>0.8\right) ; n$, number of individuals.

We estimated an effect size for BMI per minor allele of FTO to $0.32 \mathrm{~kg} \mathrm{~m}^{-2}$, which is similar to the findings in other large-scale studies in Caucasian adults, where effect sizes ranging between 0.26 and $0.39 \mathrm{~kg} \mathrm{~m}^{-2}$ have been reported (1,56-59). The corresponding effect size for WC in the present meta-analysis was $0.76 \mathrm{~cm}$ per additional minor allele, which is within the range of $0.73-1.00 \mathrm{~cm}$ per additional minor allele previously reported in large-scale studies in Caucasian adults $(1,57,60)$.

Our linear estimates of associations between BMI and mortality and between WC and mortality are probably biased because of the U- or J-shaped associations (43$46,48,49)$, but the other estimated associations shown in Table 2 are likely to reflect the expected monotonic, approximate linear associations $(45,46,48,49)$. Thus, previous studies suggest that WC has a strong positive relation to mortality when adjusted for BMI, whereas BMI adjusted for WC is inversely associated with mortality (46-48). In agreement with these relationships, a study found a direct association between fat mass and mortality and an inverse association between lean mass and mortality (45). Further, when adjusting fat mass for $\mathrm{WC}$, the positive association with mortality was eliminated, whereas adjustment of lean mass for WC did not alter the association with mortality (49).

As reported by others studying the relation between the FTO SNPs and the health-damaging effects of adiposity (5-7), the present study offers an opportunity to interpret the association of the adiposity phenotypes with all-cause mortality in the conceptual framework of so-called Mendelian randomization analysis by using the FTO SNP as an instrumental variable (61). A key requirement of these analyses is that the instrumental variable, here the alleles of the SNP, is associated with the factor to be investigated, here mortality, only through the investigated cause, here the adiposity phenotypes, i.e. there must be no pleiotropic effects. The present results support this assumption for the FTO SNP. Such analysis provides a calculation of the 
association of the adiposity phenotypes with mortality that is presumed to be unbiased by confounding or reverse causality and may hence be interpreted as evidence for a causal relation (61). For both BMI and FMI, the causal calculation for mortality is a $\mathrm{HR}$ of 1.05 per $\mathrm{kg} \mathrm{m}^{-2}$. This is greater than the observed mortality for BMI (HR of 1.02), but equal to that observed for FMI. The lower mortality observed for BMI than calculated from the instrumental variable analysis may reflect confounding and reverse causality, possibly inducing the inverse relation between the lean body mass component of BMI and mortality (45). The equality of the observed and calculated HR for FMI suggests that the observed association between FMI and mortality is probably unbiased and may reflect a causal relation.

The construction of the present study does not allow us to conduct a proper in-depth Mendelian randomization analysis (61). In spite of the size of the study, it was not originally set up to address such analysis and there remains considerable statistical uncertainty of the components used for the calculations. Furthermore, the inference would be considerably improved by various measures such as inclusion of adiposity-associated SNPs in other genomic regions, integration of the different measures of body composition and shape, calculation of the associations of the adiposity phenotypes with mortality under proper control of the well-known confounding factors (e.g. smoking), analyses taking into account non-linear relations between the adiposity phenotypes and mortality and subdivision of the mortality by age at death and by major causes of death.

The key strength of the present study is that it is strictly hypothesis-driven and designed for and capable of testing the proposed hypothesis. The meta-analysis was based on analysis of original individual participant data according to a standardized plan in all eligible cohorts. This analytical standardization across studies minimized study heterogeneity and the usage of all identified data minimized bias related to study selection, which might otherwise have caused serious publication bias because of the difficulty in publishing null results. However, as the study was conducted among Caucasians only, it is difficult to generalize the results to other ethnic groups.

We conclude that the FTO SNP is not associated with all-cause mortality independently of the adiposity phenotypes.

\section{Conflict of interest statement}

No conflict of interest was declared.

\section{Acknowledgements}

L. H. Ängquist was supported by a grant from the Danish Strategic Research Council (GENDINOB; grant number: 09-067111).
The Malmö Diet \& Cancer Study was initiated and planned in collaboration with the International Agency for Research on Cancer, the Swedish Cancer Society and Swedish Medical Research Council and the Faculty of Medicine Lund University, Sweden. The study is also funded by Region Skåne, City of Malmö, Påhlsson Foundation and the Swedish Heart and Lung Foundation.

This work of Dr. S. SMirza and Dr. H. Tiemeier is supported by the Research Institute for Diseases in the Elderly (014-93-015; RIDE2), the Netherlands Genomics Initiative (NGI)/Netherlands Consortium for Healthy Ageing (NCHA) project No. 050-060-810.

The Rotterdam Study is funded by Erasmus Medical Center, Rotterdam, the Netherlands Organization for the Health Research and Development (ZonMw), the Ministry of Education, Culture and Science and the Ministry for Health, Welfare and Sports.

The EPIC Norfolk Study is funded by programme grants from the Medical Research Council UK and Cancer Research UK and by additional support from the European Union, Stroke Association, British Heart Foundation, Department of Health, Food Standards Agency and the Wellcome Trust.

The WGHS is supported by HL043851 and HL080467 from the National Heart, Lung, and Blood Institute (NHLBI) and CA047988 from the National Cancer Institute, the Donald W. Reynolds Foundation, with collaborative scientific support and funding for genotyping provided by Amgen.

EGCUT received financing by FP7 grants (278913, 306031, 313010), Center of Excellence in Genomics (EXCEGEN) and University of Tartu (SP1GVARENG), Estonian Research Council Grant IUT20-60, the Estonian Research Roadmap through the Estonian Ministry of Education and Research and the Estonian Science Foundation (ETF9353).

The NHS and HPFS were supported by grants DK091718, HL071981, HL073168, CA87969, CA49449, CA055075, HL34594， HL088521， U01HG004399， DK080140, P30DK46200, U01CA137088, U54CA155626, DK58845, DK098311， U01HG004728， EY015473， CA134958, DK70756 and DK46200 from the National Institutes of Health (NIH), with additional support for genotyping from Merck Research Laboratories, North Wales, PA.

The Age, Gene/Environment Susceptibility (AGES) study has been funded by NIH contract N01-AG-1-2100, the NIA Intramural Research Program, Hjartavernd (the Icelandic Heart Association) and the Althingi (the Icelandic Parliament). The study is approved by the Icelandic National Bioethics Committee, VSN: 00-063. The researchers are indebted to the participants for their willingness to participate in the study.

The 1905-cohort is supported by a grant from the Danish National Research Foundation and the National 
Institute on Aging. The Danish Aging Research Center is supported by the Velux Foundation.

LURIC received funding by the 6th Framework Program (integrated project Bloodomics, grant LSHM-CT-2004503485), by the 7th Framework Program (integrated project AtheroRemo, grant agreement number 201668 and RiskyCAD, grant agreement number 305739) of the European Union, by the INTERREG IV Oberrhein Program (Project A28, Genetic mechanisms of cardiovascular diseases) with support from the European Regional Development Fund (ERDF) and the Wissenschaftsoffensive TMO.

The Health, Aging, and Body Composition (HealthABC) Study was supported in part by the Intramural Research Program of the NIH, National Institute on Aging and NIA contracts N01AG62101, N01AG62103 and N01AG62106. The genome-wide association study was funded by NIA grant 1R01AG032098-01A1 to Wake Forest University Health Sciences and genotyping services were provided by the Center for Inherited Disease Research (CIDR). CIDR is fully funded through a federal contract from the National Institutes of Health to The Johns Hopkins University, contract number HHSN268200782096C.

The British Women Heart and Health Study is supported by British Heart Foundation programme grant no. PG/13/ 66/30442 and Department of Health Policy Research Programme (0090049). Professor J. P. Casas is supported by the National Institute of Health Research University College London Hospitals Biomedical Research Centre.

The SHIP study is part of the Community Medicine Research net of the University of Greifswald, Germany, which is funded by the Federal Ministry of Education and Research (grants no. 01ZZ9603, 01ZZ0103 and 01ZZ0403), the Ministry of Cultural Affairs as well as the Social Ministry of the Federal State of Mecklenburg-West Pomerania and the network 'Greifswald Approach to Individualized Medicine (GANI_MED)’ funded by the Federal Ministry of Education and Research (grant 03IS2061A). Genome-wide data have been supported by the Federal Ministry of Education and Research (grant no. 03ZIK012) and a joint grant from Siemens Healthcare, Erlangen, Germany and the Federal State of Mecklenburg-West Pomerania. The University of Greifswald is a member of the 'Center of Knowledge Interchange' programme of the Siemens AG and the Caché Campus program of the InterSystems GmbH.

The PROSPER study was supported by an investigator initiated grant obtained from Bristol-Myers Squibb. Professor Dr. J. W. Jukema is an Established Clinical Investigator of the Netherlands Heart Foundation (grant 2001 D 032). Support for genotyping was provided by the seventh framework programme of the European Commission (grant 223004) and by the Netherlands Genomics Initiative (Netherlands Consortium for Healthy Aging grant 050-060-810).
The Caerphilly prospective study was set up by Professor Peter Elwood (MRC Unit South Wales). The DNA archive was established by a MRC (UK) grant. The Health and Social Care Information Centre (formerly the Office of National Statistics) provided the linked mortality data. The CaPS data archive is maintained by the SSCM, University of Bristol.

The Heinz Nixdorf Recall Study thank the Heinz Nixdorf Foundation (Germany), the German Federal Ministry of Research and Education (NGFN programme project \#01GS0820) and projects SI 236/8-1 and SI 236/9-1 from the German Research Council for the generous support of this study. We acknowledge the support of the Sarstedt AG \& Co. (Nümbrecht, Germany) for laboratory equipment. We are indebted to all study participants and to the dedicated personnel of the study centre of the Heinz Nixdorf Recall study. Advisory Board: Meinertz T, Hamburg, Germany (Chair); Bode C, Freiburg, Germany; de Feyter PJ, Rotterdam, Netherlands; Güntert B, Hall i.T., Austria; Gutzwiller F, Bern, Switzerland; Heinen H, Bonn, Germany; Hess O, Bern, Switzerland; Klein B, Essen, Germany; Löwel H, Neuherberg, Germany; Reiser M, Munich, Germany; Schwaiger M, Munich, Germany; Steinmüller C, Bonn, Germany; Theorell T, Stockholm, Sweden; Willich SN, Berlin, Germany.

Support for FUSION was provided by NIH grants R01DK062370 (to M. Boehnke).

The MONICA population surveys were supported by unrestricted grants from the Conseil Régional du NordPas de Calais, the Caisse Primaire d'Assurance Maladie de Sélestat, the Association Régionale de Cardiologie d'Alsace, ONIVINS, the Parke-Davis Laboratory, the Mutuelle Générale de l'Education Nationale (MGEN), the Groupe Fournier, the Réseau National de Santé Publique, the Direction Générale de la Santé, the Institut National de la Santé Et de la Recherche Médicale (INSERM), the Institut Pasteur de Lille, and the Unité d'Evaluation du Centre Hospitalier et Universitaire de Lille.

The FINCAVAS work was supported by the Competitive Research Funding of the Tampere University Hospital (Grant 9M048 and 9N035), the Finnish Cultural Foundation, the Finnish Foundation for Cardiovascular Research, the Emil Aaltonen Foundation, Finland and the Tampere Tuberculosis Foundation. The authors thank the staff of the Department of Clinical Physiology for collecting the exercise test data.

The WHII study has been supported by grants from the Medical Research Council, British Heart Foundation; Health and Safety Executive; Department of Health; NHLBI (HL036310) and National Institute on Aging (AG13196), USA, NIH; Agency for Health Care Policy Research (HS06516); and the John D and Catherine T MacArthur Foundation Research Networks on Successful Midlife Development and Socio-economic Status and 
Health. Professor MK's time on this manuscript was partially supported by the (HL36310).

PREVEND genetics is supported by the Dutch Kidney Foundation (Grant E033), the NIH (grant LM010098), The Netherlands Organization for Scientific Research (NWO-Groot 175.010.2007.006, NWO VENI grant 916.761.70 and NWO VIDI grant 917.13.350, ZonMW grant 90.700.441) and the Dutch Inter University Cardiology Institute Netherlands. N. Verweij is supported by the Netherlands Heart Foundation (grant NHS2010B280).

The KORA research platform (KORA, Cooperative Research in the Region of Augsburg) was initiated and financed by the Helmholtz Zentrum München - German Research Center for Environmental Health, which is funded by the German Federal Ministry of Education and Research and by the State of Bavaria. Furthermore, KORA research was supported within the Munich Center of Health Sciences (MC Health), Ludwig-MaximiliansUniversität, as part of LMUinnovativ.

The SHEEP study was supported by the Swedish Council for Social Research, the Swedish Council for Work Life Research, the Stockholm County Council, the Swedish Heart and Lung Foundation and the Swedish Research Council.

The CoLaus study was supported by research grants from GlaxoSmithKline, the Faculty of Biology and Medicine of Lausanne, Switzerland and four grants of the Swiss National Science Foundation (grants \#3200B0-105993, \#3200B0-118308, \#33CSCO-122661 and 33CS30139468) and thankfully acknowledges Diana Marek's contributions to this study.

The MRC NSHD study was funded by the Medical Research Council (MC_UU_12019/1).

We thank all EPIC-Potsdam participants for their invaluable contribution to the study. Furthermore, we thank Ellen Kohlsdorf for data management as well as the follow-up team headed by Dr. Manuala Bergmann. The recruitment phase of the EPIC-Potsdam study was supported by the Federal Ministry of Science, Germany (01 EA 9401) and the European Union (SOC 9520140805 F02). The follow-up of the EPIC-Potsdam study was supported by German Cancer Aid (70-2488-Ha I) and the European Community (SOC 9820076905 F02).

The Australian Twin-Family Study (AUSTWIN) acknowledge funding from the Australian National Health and Medical Research Council (NHMRC grants 241944, 389875, 389891, 389892, 389938, 442915, 442981, 496739 and 552485), US NIH (NIH grants AA07535, AA10248 and AA014041) and the Australian Research Council (ARC grant DP0770096). P. M. Ridker was supported by an NHMRC Career Development Award.

Helsinki Birth Cohort Study has been supported by grants from the Academy of Finland (Grant No. 120386 and 125876 to J. G. Eriksson), the Finnish Diabetes
Research Society, Novo Nordisk Foundation, Finska Läkaresällskapet, Liv och Hälsa, Samfundet Folkhälsan, Finska Läkaresällskapet and the Signe and Ane Gyllenberg foundation.

The Danish Diet, Cancer and Health (DCH) cohort is supported by the Danish Cancer Society.

The Finnish Diabetes Prevention Study was funded by the Academy of Finland, Nord Forsk and Nordic Centre of Excellent Sysdiet; furthermore, contributions from the Finnish Diabetes Prevention Study group and especially Markku Peltonen and Professor Matti Uusitupa are gratefully acknowledged.

The SAVITAIPALE study received grant support from the US NIH (DK62370)

The IMPROVE study was supported by the European Commission (Contract number: QLG1-CT-2002-00896), Swedish Heart-Lung Foundation, Swedish Research Council (projects 8691 and 0593), Knut and Alice Wallenberg Foundation, Foundation for Strategic Research, Stockholm County Council (project 592229), Strategic Cardiovascular and Diabetes Programmes of Karolinska Institutet and Stockholm County Council, European Union Framework Programme 7 (FP7/2007-2013) for Innovative Medicine Initiative ( ${ }^{\circ}$ IMI/115006 [the SUMMIT consortium]), Magnus Bergwall Foundation, Academy of Finland (Grant \#110413), British Heart Foundation (RG2008/08, RG2008/014) and Italian Ministry of Health (Ricerca Corrente). R. J. Strawbridge is supported by the Swedish Heart-Lung Foundation, the Tore Nilsson, Gamla Tjänarinnor and Fredrik and Ingrid Thurings foundations.

The GEMINAKAR project was supported by grants from the Danish Medical Research Council, the Danish Diabetes Association, the NOVO Foundation, the Danish Heart Foundation, Apotekerfonden, the Foundation of A. and J. Louis-Hansen, the Foundation of Direktor E. Danielsen and wife, the Foundation of Direktor K. Bonnelycke and wife Grethe, the Foundation of Laegevidenskabens Fremme, the Foundation of A. F. Bolding, the Foundation of O. William and E. B. Olesen, the Faculty of Health at University of Southern Denmark, the Danish National Science Foundation, T. Steenbeck's Foundation, the Gangsted Foundation and King Christian the Tenth's Foundation. K. O. Kyvik received funding from the European Union Contract No. QLG2-CT-2002-01254 (the GenomEUtwin project). The Danish Epidemiology Science Centre is supported by the National Danish Science Foundation. Finally, we would like to thank all twins participating in the GEMINAKAR Study.

\section{Supporting information}

Additional Supporting Information may be found in the online version of this article, http://dx.doi.org/10.1111/ obr. 12263 
Appendix S1. Note on calculation of the association between adiposity traits and mortality by Mendelian randomization based on the FTO SNP as instrumental variable

Table S1. Descriptive information of the 37 study samples included in the meta-analysis sorted alphabetically by study name.

Table S2. Association of adiposity phenotypes and FTO on all-cause mortality using age restrictions to being older than 60, 65 and 70 years, respectively.

Table S3. Association of adiposity phenotypes and FTO on all-cause mortality separately for males and females.

\section{References}

1. Frayling TM, Timpson NJ, Weedon MN et al. A common variant in the FTO gene is associated with body mass index and predisposes to childhood and adult obesity. Science 2007; 316: 889-894.

2. Dina C, Meyre D, Gallina S et al. Variation in FTO contributes to childhood obesity and severe adult obesity. Nat Genet 2007; 39: 724-726.

3. Scuteri A, Sanna S, Chen WM et al. Genome-wide association scan shows genetic variants in the FTO gene are associated with obesity-related traits. PLoS Genet 2007; 3: e115.

4. Kring SI, Holst C, Zimmermann E et al. FTO gene associated fatness in relation to body fat distribution and metabolic traits throughout a broad range of fatness. PLOS ONE 2008; 3: e2958.

5. Nordestgaard BG, Palmer TM, Benn M et al. The effect of elevated body mass index on ischemic heart disease risk: causal estimates from a Mendelian randomisation approach. PLoS Med 2012; 9: e1001212.

6. Timpson NJ, Harbord R, Davey SG, Zacho J, Tybjaerg-Hansen A, Nordestgaard BG. Does greater adiposity increase blood pressure and hypertension risk?: mendelian randomization using the FTO/MC4R genotype. Hypertension 2009; 54: 84-90.

7. Timpson NJ, Nordestgaard BG, Harbord RM et al. C-reactive protein levels and body mass index: elucidating direction of causation through reciprocal Mendelian randomization. Int J Obes (Lond) 2011; 35: 300-308.

8. Davey SG, Sterne JA, Fraser A, Tynelius P, Lawlor DA, Rasmussen F. The association between BMI and mortality using offspring BMI as an indicator of own BMI: large intergenerational mortality study. BMJ 2009; 339: b5043.

9. Zimmermann E, Kring SI, Berentzen TL et al. Fatnessassociated FTO gene variant increases mortality independent of fatness - in cohorts of Danish men. PLoS ONE 2009; 4: e4428. 10. Harris TB, Launer LJ, Eiriksdottir G et al. Age, gene/ environment Susceptibility-Reykjavik study: multidisciplinary applied phenomics. Am J Epidemiol 2007; 165: 1076-1087.

11. Benyamin B, Ferreira MA, Willemsen G et al. Common variants in TMPRSS6 are associated with iron status and erythrocyte volume. Nat Genet 2009; 41: 1173-1175.

12. Lawlor DA, Bedford C, Taylor M, Ebrahim S. Geographical variation in cardiovascular disease, risk factors, and their control in older women: British Women's Heart and Health Study. J Epidemiol Community Health 2003; 57: 134-140.

13. Yarnell JWG, Elwood PC, Sweetnan PM. Caerphilly and Speedwell collaborative heart disease studies. The Caerphilly and
Speedwell Collaborative Group. I Epidemiol Community Health 1984; 38: 259-262.

14. Firmann M, Mayor V, Vidal PM et al. The CoLaus study: a population-based study to investigate the epidemiology and genetic determinants of cardiovascular risk factors and metabolic syndrome. BMC Cardiovasc Disord 2008; 8: 6.

15. Nybo H, Gaist D, Jeune B et al. The Danish 1905 cohort: a genetic-epidemiological nationwide survey. J Aging Health 2001; 13: $32-46$.

16. Tjonneland A, Olsen A, Boll K et al. Study design, exposure variables, and socioeconomic determinants of participation in Diet, Cancer and Health: a population-based prospective cohort study of 57,053 men and women in Denmark. Scand J Public Health 2007; 35: 432-441.

17. Leitsalu L, Haller T, Esko T et al. Cohort Profile: Estonian Biobank of the Estonian Genome Center, University of Tartu. Int J Epidemiol 2014 [Epub ahead of print].

18. Day N, Oakes S, Luben R et al. EPIC-Norfolk: study design and characteristics of the cohort. European Prospective Investigation of Cancer. Br J Cancer 1999; 80(Suppl. 1): 95-103.

19. Boeing H, Wahrendorf J, Becker N. EPIC-Germany - A source for studies into diet and risk of chronic diseases. European Investigation into Cancer and Nutrition. Ann Nutr Metab 1999; 43: 195-204.

20. Nieminen T, Lehtinen R, Viik J et al. The Finnish Cardiovascular Study (FINCAVAS): characterising patients with high risk of cardiovascular morbidity and mortality. BMC Cardiovasc Disord 2006; 6: 9.

21. Eriksson J, Lindstrom J, Valle T et al. Prevention of Type II diabetes in subjects with impaired glucose tolerance: the Diabetes Prevention Study (DPS) in Finland. Study design and 1-year interim report on the feasibility of the lifestyle intervention programme. Diabetologia 1999; 42: 793-801.

22. Scott LJ, Mohlke KL, Bonnycastle LL et al. A genome-wide association study of type 2 diabetes in Finns detects multiple susceptibility variants. Science 2007; 316: 1341-1345.

23. Benyamin B, Sørensen TIA, Schousboe K, Fenger M, Visscher PM, Kyvik KO. Are there common genetic and environmental factors behind the endophenotypes associated with the metabolic syndrome? Diabetologia 2007; 50: 1880-1888.

24. Yliharsila H, Kajantie E, Osmond C, Forsen T, Barker DJ, Eriksson JG. Birth size, adult body composition and muscle strength in later life. Int J Obes (Lond) 2007; 31: 13921399 .

25. Schmermund A, Mohlenkamp S, Stang A et al. Assessment of clinically silent atherosclerotic disease and established and novel risk factors for predicting myocardial infarction and cardiac death in healthy middle-aged subjects: rationale and design of the Heinz Nixdorf RECALL Study. Risk Factors, Evaluation of Coronary Calcium and Lifestyle. Am Heart J 2002; 144: 212218.

26. Rimm EB, Stampfer MJ, Colditz GA, Giovannucci E, Willett WC. Effectiveness of various mailing strategies among nonrespondents in a prospective cohort study. Am J Epidemiol 1990; 131: 1068-1071.

27. Baldassarre D, Nyyssonen K, Rauramaa R et al. Crosssectional analysis of baseline data to identify the major determinants of carotid intima-media thickness in a European population: the IMPROVE study. Eur Heart J 2010; 31: 614-622.

28. Holle R, Happich M, Lowel H, Wichmann HE. KORA - a research platform for population based health research. Gesundheitswesen 2005; 67(Suppl. 1): S19-S25.

29. Winkelmann BR, Marz W, Boehm BO et al. Rationale and design of the LURIC study - a resource for functional genomics, 
pharmacogenomics and long-term prognosis of cardiovascular disease. Pharmacogenomics 2001; 2: S1-S73.

30. Manjer J, Carlsson S, Elmstahl S et al. The Malmo Diet and Cancer Study: representativity, cancer incidence and mortality in participants and non-participants. Eur J Cancer Prev 2001; 10: 489-499.

31. Stewart AW, Kuulasma K, Beaglehole R. Ecological analysis of the association between mortality and major risk factors of cardiovascular disease. The World Health Organization MONICA Project. Int J Epidemiol 1994; 23: 505-516.

32. Colditz GA, Manson JE, Hankinson SE. The Nurses' Health Study: 20-year contribution to the understanding of health among women. J Womens Health 1997; 6: 49-62.

33. Rousseau K, Vinall LE, Butterworth SL et al. MUC7 haplotype analysis: results from a longitudinal birth cohort support protective effect of the MUC7*5 allele on respiratory function. Ann Hum Genet 2006; 70: 417-427.

34. Wadsworth M, Kuh D, Richards M, Hardy R. Cohort Profile: The 1946 National Birth Cohort (MRC National Survey of Health and Development). Int J Epidemiol 2006; 35: 49-54.

35. Pinto-Sietsma SJ, Janssen WM, Hillege HL, Navis G, De ZD, De Jong PE. Urinary albumin excretion is associated with renal functional abnormalities in a nondiabetic population. J Am Soc Nephrol 2000; 11: 1882-1888.

36. Shepherd J, Blauw GJ, Murphy MB et al. The design of a prospective study of Pravastatin in the Elderly at Risk (PROSPER). PROSPER Study Group. PROspective Study of Pravastatin in the Elderly at Risk. Am J Cardiol 1999; 84: 1192-1197.

37. Hofman A, Darwish MS, van Duijn CM et al. The Rotterdam Study: 2014 objectives and design update. Eur J Epidemiol 2013; 28: 889-926.

38. Saramies J. Risk factors of type 2 diabetes and screening of abnormal glucose metabolism in primary health care. Ph.D. thesis. Acta Univ Oul 2004; D812.

39. Reuterwall C, Hallqvist J, Ahlbom A et al. Higher relative, but lower absolute risks of myocardial infarction in women than in men: analysis of some major risk factors in the SHEEP study. The SHEEP Study Group. J Intern Med 1999; 246: 161-174.

40. Volzke H, Alte D, Schmidt CO et al. Cohort profile: the study of health in Pomerania. Int J Epidemiol 2011; 40: 294-307.

41. Ridker PM, Chasman DI, Zee RY et al. Rationale, design, and methodology of the Women's Genome Health Study: a genomewide association study of more than 25,000 initially healthy American women. Clin Chem 2008; 54: 249-255.

42. Marmot M, Brunner E. Cohort profile: the Whitehall II study. Int J Epidemiol 2005; 34: 251-256.

43. Berrington de Gonzalez A, Hartge P, Cerhan JR et al. Bodymass index and mortality among 1.46 million white adults. N Engl J Med 2010; 363: 2211-2219.

44. Prospective Studies Collaboration, Whitlock G, Lewington S et al. Body-mass index and cause-specific mortality in 900,000 adults: collaborative analyses of 57 prospective studies. Lancet 2009; 373: 1083-1096.
45. Bigaard J, Frederiksen K, Tjonneland A et al. Body fat and fat-free mass and all-cause mortality. Obes Res 2004; 12: 10421049.

46. Bigaard J, Tjonneland A, Thomsen BL, Overvad K, Heitmann BL, Sørensen TIA. Waist circumference, BMI, smoking, and mortality in middle-aged men and women. Obes Res 2003; 11: 895903.

47. Cerhan JR, Moore SC, Jacobs EJ et al. A pooled analysis of waist circumference and mortality in 650,000 adults. Mayo Clin Proc 2014; 89: 335-345.

48. Pischon T, Boeing H, Hoffmann $\mathrm{K}$ et al. General and abdominal adiposity and risk of death in Europe. N Engl J Med 2008; 359: 2105-2120.

49. Bigaard J, Frederiksen K, Tjonneland A et al. Waist circumference and body composition in relation to all-cause mortality in middle-aged men and women. Int J Obes (Lond) 2005; 29: 778784.

50. Schoenfeld D. Chi-squared goodness of fit tests for the proportional hazards regression model. Biometrika 1980; 67: 145153.

51. Borenstein M, Hedges L, Higgins J, Rothstein H. Introduction to Meta-Analysis. John Wiley \& Sons, Ltd: West Sussex, 2009.

52. DerSimonian R, Laird N. Meta-analysis in clinical trials. Control Clin Trials 1986; 7: 177-188.

53. Sterne JAC (ed). Meta-Analysis in Stata: An Updated Collection from the Stata Journal. Stata Press: College Station, TX, 2009. 54. Higgins JP, Thompson SG, Deeks JJ, Altman DG. Measuring inconsistency in meta-analyses. BMJ 2003; 327: 557-560.

55. Liu C, Mou S, Pan C. The FTO gene rs9939609 polymorphism predicts risk of cardiovascular disease: a systematic review and meta-analysis. PLoS ONE 2013; 8: e71901.

56. Hertel JK, Johansson S, Sonestedt E et al. FTO, type 2 diabetes, and weight gain throughout adult life: a meta-analysis of 41,504 subjects from the Scandinavian HUNT, MDC, and MPP studies. Diabetes 2011; 60: 1637-1644.

57. Li H, Kilpelainen TO, Liu C et al. Association of genetic variation in FTO with risk of obesity and type 2 diabetes with data from 96,551 East and South Asians. Diabetologia 2012; 55: $981-$ 995.

58. Loos RJ, Lindgren CM, Li S et al. Common variants near MC4R are associated with fat mass, weight and risk of obesity. Nat Genet 2008; 40: 768-775.

59. Thorleifsson G, Walters GB, Gudbjartsson DF et al. Genomewide association yields new sequence variants at seven loci that associate with measures of obesity. Nat Genet 2009; 41: 18-24. 60. Heard-Costa NL, Zillikens MC, Monda KL et al. NRXN3 is a novel locus for waist circumference: a genome-wide association study from the CHARGE Consortium. PLoS Genet 2009; 5: e1000539.

61. Lawlor DA, Harbord RM, Sterne JA, Timpson N, Davey SG. Mendelian randomization: using genes as instruments for making causal inferences in epidemiology. Stat Med 2008; 27: 11331163. 